\title{
EDUCACIÓN INFANTIL DE CALIDAD EN ESTADOS UNIDOS
}

\section{High quality early childhood education in the United}

\section{States}

\section{Mercedes Varona Alabern*}

\section{RESUMEN}

El sistema norteamericano de educación, representado por las siglas K-12 cosechó impresionantes frutos durante gran parte del siglo pasado. Sus orígenes se remontan a principios del siglo XX, cuando las necesidades sociales y económicas del país distaban ampliamente de las presentes. Nuestra sociedad actual, mucho más compleja, demanda mayor preparación de la ciudadanía, la cual se cimienta, como demostraremos en este artículo, con una educación infantil de calidad. Por este motivo, la mayoría de especialistas en educación reclaman a las administraciones públicas la creación de un sistema de educación infantil universal y verdaderamente de calidad (no meras guarderías de la infancia) para lograr aumentar la preparación de la población norteamericana, mejorar la economía y eliminar las enquistadas desigualdades sociales.

PALABRAS CLAVE: Educación Infantil, Calidad Educativa, Estados Unidos, Diferencias Académicas, Ratio Alumno-Profesor.

\section{$\underline{\text { ABSTRACT }}$}

The United States education system, K-12 produced impressive results for most of the 20th century. We trace its origins back to the end of the 19th century, when the America's social and economical needs and its circumstances were very different from the

* Universidad de Valencia (España). 
ones today. Nowadays, American society is far more complex and demands highly qualified citizens, a requirement which inevitably starts with high quality early childhood education, as we will illustrate below. Therefore, experts are currently requesting all public administrations and private sector to take further steps to ensure high quality preschool education for everyone in order to improve Americans' skills and the United States' economy, as well as to overcome the existing inequalities.

KEY WORDS: Early Childhood Education, High Quality Education, United States, Achievement Gap, Teacher/Student Ratios in Pre-School.

$* * * * *$

\section{INTRODUCCIÓN}

Estados Unidos ha sido pionero en escolarizar a su población de forma universal y gratuita, adelantándose claramente a su época y obteniendo suculentos réditos sociales y económicos durante todo el siglo XX. No obstante, el entusiasmo generalizado de la formación de la población que impregnó la vida social y política de la primera mitad del siglo pasado, fue reduciéndose con el paso del tiempo. El sistema educativo norteamericano actual fue establecido de forma oficial para toda la nación por la administración Roosevelt en 1965: K-12. Sin embargo, el sistema educativo norteamericano, competencia exclusiva de las administraciones estatales y locales, ya había cosechado grandes éxitos antes de la promulgación de dicha ley federal. Ello, sumado a las diferencias académicas que aparecieron durante el mismo periodo, pusieron de manifiesto la necesidad de la entrada en escena del gobierno de la nación, a través de una ley homogénea y redistribuidora dirigida a todos los centros escolares. Después de más de 50 años de vigencia de esta ley federal y de las promesas electorales a favor de una educación infantil de calidad, en plena era de la información y de la globalización, el innovador y vanguardista sistema educativo federal norteamericano de 1965 se ha quedado obsoleto especialmente en lo referente a la educación infantil, avalado por la abrumadora evidencia de la necesidad de ofrecer educación infantil de calidad de forma universal para asentar las bases de la formación de la ciudadanía.

2. IMPERANTE NECESIDAD DE UNA EDUCACIÓN INFANTIL UNIVERSAL DE CALIDAD

Existen numerosos estudios sobre los beneficios de la educación infantil de calidad para el individuo, para la economía y para la sociedad, no obstante, los estados, los distritos escolares y el propio Congreso norteamericano continúan evitando su financiación, porque la inversión realizada en esta etapa educativa no se recupera a corto plazo, sino cuando muchos de estos dirigentes hayan sido renovados y sus frutos recogidos por otros políticos. 
El sector privado, por su parte, se resiste asimismo a invertir en ella, ya que persigue rendimientos instantáneos, que en esta etapa educativa no puede lograr. Esta ahorrativa actitud de la iniciativa pública y privada norteamericana contrasta con las generosas inversiones que tanto el gobierno federal, como el sector privado realizan en educación superior porque sus resultados económicos se materializan a corto plazo. El gasto total estimado en educación en EEUU durante 2013 alcanzó los \$1.165.000 millones. La educación K-12 absorbió $\$ 659.000$ millones (un 56,6\%) de esos fondos para atender a 52 millones de escolares ( $\$ 12.673$ por alumno), con una participación del gobierno federal en la educación pública del 12,5\% (incrementándose del 9\% aproximado antes de la recesión económica). Por su parte, $\$ 496.000$ millones se destinaron a la educación superior (representando el 42,6\% del total) para atender a unos 28 millones de alumnos (quienes no necesariamente estudiaron a tiempo completo, como lo hicieran los escolares de las etapas K-12), ascendiendo a $\$ 17.714$ el gasto por alumno y alcanzando la financiación federal el $17 \%$ de la educación superior pública ${ }^{1}$ Asimismo, la inversión privada para las etapas K-12 representó el 7\% de los gastos, contrastando con el 37,5\% invertido por los fondos privados en la educación superior (U.S. DEPARTMENT OF EDUCATION, 2013)

Numerosos expertos han defendido y avalado la necesidad de una educación infantil universal de calidad desde hace años, intensificándose durante las últimas décadas, como se desprende a continuación.

Según el premio Nobel de Economía, Laureate James Heckman de la Universidad de Chicago (2004), el 50\% de las diferencias académicas entre los alumnos de high school (bachillerato o similar) ya estaban presentes antes de comenzar la enseñanza obligatoria y gratuita a los 5 años. El éxito o fracaso escolar de los estudiantes tiene sus orígenes en la educación infantil (HECKMAN y MASTEROV, 2004; CLEMENT, 2005).

El profesor Heckman (2012) demuestra asimismo que para que la economía de EEUU continúe siendo puntera, la población infantil, que vive en situaciones adversas (pobreza, abusos, etc.), debe escolarizarse en programas educativos de calidad. Estos programas no sólo mejoran los resultados académicos, sino que tienen un efecto muy positivo en las habilidades no-cognitivas, como motivación, esfuerzo, etc., que a la larga son los recursos más importantes en la vida del ser humano. Añade, que aquellos programas que apoyan la infancia y las familias en riesgo reducen la delincuencia en un $70 \%$ durante los 20 años siguientes. Por consiguiente, concluye que "la educación infantil no sólo es moralmente necesaria, sino que es rentable para la sociedad".

Timothy J. Bartik (2006) considera que las subvenciones estatales para atraer empresas aportan mayores beneficios a corto y medio plazo (hasta 15 años) que las

\footnotetext{
1 Los fondos federales también financian la educación superior privada oscilando entre 15\%, para instituciones sin ánimo de lucro y 6\%, para instituciones con ánimo de lucro.
} 
inversiones en educación infantil de calidad, pero a largo plazo (30 años) estas últimas crean un mayor número de puestos de trabajo a nivel nacional $(1,3 \%$, frente al $0,6 \%)$. A esto hay que añadir que las empresas se mueven de un estado a otro buscando la mayor subvención. Los estados obtienen 3 dólares por cada dólar invertido en educación infantil de calidad y el gobierno federal, 4 dólares. Una educación infantil de calidad en EEUU crearía 3 millones de nuevos puestos de trabajo, aumentando los ingresos en $\$ 300.000$ millones, el Producto Interior Bruto (PIB) crecería casi un billón de dólares y las recaudaciones tributarias en $\$ 200.000$ millones durante los siguientes 75 años (cifra basada en la esperanza de vida norteamericana en 2003 situada en 78 años).

Rolnick y Grunewald (2003) coinciden con Bantik, cuestionando las subvenciones a empresas para atraer negocios a zonas con altos niveles de desempleo, ya que los negocios se trasladan en función de las subvenciones y los trabajadores las siguen. De esta forma, se genera empleo en un lugar y se pierde en otro. En cambio, si la financiación estatal fuera dirigida a crear una educación infantil de calidad, los beneficios serían para todos, logrando un rendimiento interno del $16 \%$ ( $12 \%$ para la comunidad y $4 \%$ para el individuo). Concluyen exhortando a las administraciones públicas a subvencionar la educación infantil de calidad por carecer de los fondos públicos y privados necesarios.

En 2005, la asociación The RAND realizó un estudio sobre los costes y los beneficios que aportarían los programas infantiles de 4 años de calidad en el estado de California (Estos programas tendrían un máximo de 20 niños por clase con 2 profesores altamente cualificados). La educación infantil de calidad que esta asociación propone costaría a las arcas estatales $\$ 4.300$ adicionales por niño, pero el beneficio bruto alcanzaría los \$13.700 por escolar, es decir, 3,15 dólares por cada dólar invertido. El estado y las administraciones locales se beneficiarían del $50 \%$ de estas cantidades. Este estudio puso de manifiesto además que los niños pertenecientes a las clases medias y medias bajas eran el colectivo menos representado en la educación infantil de calidad en el estado de California. Por todo ello, este informe señala que una formación preescolar universal de calidad beneficiaría también a estas clases sociales, al disminuir el fracaso escolar, las retenciones, las clases de refuerzo, etc., demostrando que el coste se recuperaría cuando los estudiantes cumplieran 14 años.

La asociación para la investigación sobre la educación infantil, National Institute for Early Education Research, a través de Belfield y Schwartz (2006), demostró que la educación infantil de calidad mejoraba los rendimientos académicos de los escolares y el comportamiento de los alumnos en los años venideros, reduciendo además el fracaso escolar, la violencia, el consumo de drogas, etc. Por cada niño de 4 años escolarizado en un programa de calidad, el estado ahorraría entre $\$ 2.600$ y $\$ 4.400$ durante todo el periodo escolar. 
Dickens, Sawhill, y Tebbs (2006) señalaron que los niños escolarizados en educación infantil de calidad tenían mayor probabilidad de terminar sus estudios obligatorios y obtener titulaciones superiores, lo que se traducía en una mayor productividad laboral y por tanto, en un mayor Producto Interior Bruto (PIB). Estimaban que el coste de la educación infantil universal de calidad alcanzaría los \$59.000 millones, comenzando a recuperar dicha inversión en 2050 y para 2080, los ingresos habrían doblado los costes.

Desde la Universidad de Minnesota (REYNOLS, TEMPLE, OU, ARTEGA, y WHITE, 2011; REYNOLDS, 2011) se llevó a cabo un proyecto que consistió en realizar un seguimiento a 1.400 niños de 3 años en educación infantil, hasta que cumplieron 25 años. Los resultados evidenciaron una vez más, la importancia de una educación infantil de calidad, ya que el grupo que había recibido una formación de calidad durante su infancia había logrado mejor posicionamiento socioeconómico, mejor salud física y menor probabilidad de consumir sustancias tóxicas y de delinquir. Paralelamente, señalan que la educación infantil de calidad disminuye las necesidades de educación especial, de apoyo o de adaptación curricular.

El programa Child Parent Center (CPC) (PROMISING PRACTICES NETWORK, 2008; REYNOLS, 2011 y 1999; REYNOLDS, TEMPLE, ROBERTSON y MANN, 2001; MUELLER, et ál, 1974) nació en 1967 en los barrios más deprimidos de Chicago, gracias a los fondos federales procedentes del Title I (Capítulo I) de la ley de enseñanza Elementary and Secondary Education Act de 1965 (ESEA). Dicho programa, todavía vigente en la actualidad, destaca por poseer un profesorado altamente cualificado (con licenciaturas y especialidades en enseñanza infantil). Atiende a escolares entre 3 y 4 años y en ocasiones hasta los primeros años de primaria. Los ratios se mantienen por debajo de 17 alumnos por cada 2 profesores y la enseñanza se centra en las materias consideradas fundamentales (lectura y matemáticas). La docencia es acompañada de visitas regulares a domicilio por parte del profesorado, así como, de seguimiento nutricional y sanitario. Los resultados del estudio, realizado desde 1979 a 1989 niños de 1 año de edad hasta que cumplieron los 21 años, pusieron de manifiesto la efectividad del mismo, al compararlos con el grupo de control, tal como se detalla a continuación:

- Obtuvieron mejores resultados académicos en materias de lectura y de matemáticas en el $8^{\circ}$ curso de la etapa educativa norteamericano representado por K-12.

- Sufrieron menor probabilidad de necesitar refuerzo académico y disfrutaron de mayores posibilidades de finalizar sus estudios escolares.

- La tasa de delincuencia fue considerablemente inferior y fueron menos proclives a sufrir abusos y abandono.

- Se redujo el fracaso escolar.

- Se aumentó el número de titulados de high school. 
- Se incrementó el empleo y el sueldo percibido.

Por cada dólar invertido en el programa, se obtienen $\$ 11$ a lo largo de la vida del estudiante, es decir, un $18 \%$ de rendimiento interno. Los beneficios obtenidos por la escolarización de los 100.000 alumnos del programa en las últimas décadas ascendieron a \$2.600 millones. Según los autores de este estudio, si se escolarizara a los 2 millones de niños norteamericanos de 3 y 4 años que viven bajo del umbral de la pobreza, los estados deberían realizar una inversión total de $\$ 13.200$ millones o $\$ 8.200$ millones sobre los presupuestos dedicados a educación infantil de 2008 (es decir, un 164\% más del gasto vigente), no obstante, el beneficio obtenido por esta inversión sería de $\$ 315.000$ hasta 2050 . Los resultados, obviamente, no se materializarían a corto plazo, ya que los individuos deben llegar a la edad adulta, pero durante la edad escolar de primaria y secundaria estos beneficios ya aliviarían y mejorarían el sistema educativo del país, repercutiendo en el resto de los alumnos, en el profesorado, etc. Los autores destacan que si la enseñanza infantil de calidad se hiciera disponible a todos los niños norteamericanos de 3 y 4 años, abarcaría a 7 millones según los datos de 2008, costando al erario público $\$ 43.000$ millones o $\$ 33.000$ millones más que la inversión actual. (330\% más), pero el beneficio obtenido hasta 2050 sería de $\$ 779.000$ millones, muy superior a la inversión realizada. Los datos aportados por este estudio son más persuasivos que los presentados por Dickens, Sawhill, y Tebbs (2006), pero ambos tienen el mismo objetivo.

Carolina Abecedarian Program: El programa Carolina Abecedarian Project nació en 1972, con 57 niños de 3 meses de edad. Las madres eran solteras, menores de 20 años, de etnia negra, vivían con familiares y no habían completado los estudios de high school. Los niños en este programa recibieron de 5 a 8 horas de educación pre-escolar a la semana y transporte gratuito del hogar a la escuela, mientras que los niños del grupo control recibieron atención médica, social y alimentaria para que estos factores no influyeran en el estudio. El ratio de profesor alumno era de 1 docente por 3 bebés, aumentando con la edad hasta alcanzar 1 profesor por 6 niños. Los maestros trabajaban 4 áreas: Capacidad cognitiva y coordinación; Habilidades sociales y autonomía; Lenguaje; y Habilidades motoras. A los 3 años los niños habían sido expuestos a conocimientos diversos como música, matemáticas o ciencias. Se realizó un seguimiento hasta los 21 años, a través de distintos estudios sobre el mismo programa, llegando todos ellos a resultados similares. El programa Carolina Abecedarian logró un mayor número de universitarios, una disminución del consumo de drogas, una mejor preparación en lectura, mayores habilidades profesionales, y un descenso de los embarazos durante la adolescencia (este dato es muy elevado en colectivos menos afortunados favorecido por las importantes subvenciones y ayudas públicas que tradicionalmente las madres solteras de estos segmentos de la población han recibido). El coste total del programa por alumno durante los 5 años fue de $\$ 63.476$, un elevado coste, no obstante, el beneficio estimado alcanzó los \$95.000 (THE CAROLINA ABECEDARIAN PROGRAM; CAMPBELL y RAMEY, 2007). 
Perry Preschool Program (SCHWEINHART, et al., 2005; SCHWEINHART, et al., 2006) Los niños de la ciudad de Ypsilanti en el estado de Michigan, que participaron en este programa, iniciado en 1962 y finalizado 5 años más tarde, fueron seguidos hasta cumplir los 40 años, convirtiéndolo en el estudio más largo realizado hasta la fecha. Los 58 niños de 3 y 4 años de etnia negra y en situación de alto riesgo (pobreza, bajo nivel intelectual de los progenitores y familiares, etc.), se reunían 2 horas y media diarias para trabajar intensamente las áreas intelectuales, sociales y físicas. El programa tenía una duración de 2 años y los profesores estaban altamente cualificados, manteniendo un ratio que no podía exceder de 7 alumnos por profesor. Paralelamente, la instrucción directa se complementaba con visitas semanales de 1,5 horas del docente a las familias. Los resultados de dicho estudio desvelaron beneficios significativos:

- Los niños participantes en este programa obtuvieron mejores resultados en lengua y en los test de inteligencia hasta la edad de 7 años (37\% frente al 28\%) y mejores resultados académicos a los 14 años que el grupo de control ( $45 \%$ frente al 15\%).

- Necesitaron menos educación especial y completaron sus estudios hasta el high school en un porcentaje superior que el grupo de control (77\% frente al $60 \%$.).

- A la edad de 40 años el porcentaje de estos adultos con un empleo $(60 \%$ frente a $55 \%$ ) y con mejor sueldo ( $76 \%$ frente a $62 \%$ ), fue superior que el grupo de control.

- Menor probabilidad de delinquir (36\%, frente al 55\%).

- Necesitaron menos ayudas sociales.

- Cuantificando todos estos beneficios, se obtuvieron unos ingresos adicionales de $\$ 195.621$ por niño (\$12,90 por cada dólar gastado), derivados de:

- Descenso de la delincuencia, representado un ahorro de \$171.472.

- Un aumento de la recaudación tributaria en $\$ 14,078$.

- Ahorros en necesidades educativas especiales de \$7.303.

- Ahorro en prestaciones sociales de $\$ 2.768$.

- Ingresos adicionales de $\$ 49.190$.

- Por tanto, el rendimiento total de la inversión en educación infantil ascendió a 16,14 dólares por cada dólar invertido (\$244.811/\$15.166). No fue un programa económico, ya que el coste por niño ascendió a $\$ 15.166$, no obstante, el retorno en la inversión fue muy superior.

Los estudios realizados por Gillian y Zigler (2004) indican que la educación infantil de calidad disminuye la probabilidad de repetir curso. Los participantes en estos programas obtienen mejores resultados en $4^{\circ}$ curso de primaria en las materias fundamentales, como matemáticas o lengua, base del aprendizaje del alumno. El economista Robert Lynch (2007) se unió a la férrea defensa de la educación infantil de 4 años de calidad, señalando 
que se autofinancia después de 9 años y produce una creciente rentabilidad en los años posteriores.

Todas estas investigaciones son una muestra de la extensa literatura existente sobre la incalculable aportación de la educación infantil de calidad. A esto hay que añadir los avances en el estudio del desarrollo cerebral durante la infancia. Todos ellos nos indican que el ser humano nace con un cerebro por desarrollar, por consiguiente, las experiencias en la infancia lo moldean para adquirir habilidades dirigidas al aprendizaje, a la sociabilidad, a la afectividad, a la accesibilidad laboral, al fortalecimiento de la salud física y a la felicidad. El cerebro humano empieza a desarrollarse durante las primeras semanas de gestación y continúa hasta la edad adulta. Los primeros años de vida, desde el útero materno, son fundamentales, al ser el crecimiento cerebral progresivo e interrelacionado. Por tanto, si el desarrollo cerebral es entorpecido o dificultado por circunstancias externas (carencia de estímulos externos, condiciones sanitarias y alimentarias deficientes, carencias afectivas, abusos, violencia, etc.), éstas repercuten y condicionan negativamente el aprendizaje, la sociabilidad y el equilibrio emocional y psíquico del niño, que se magnifican al llegar a la edad adulta, creando serios problemas. Sin una base fuerte para poder sostener su vida, los niños, y especialmente aquellos más vulnerables irán retrasados mucho antes de comenzar la escolarización obligatoria a los 5 años de edad (THE CENTER ON THE DEVELOPING CHILD, 2011).

A pesar de toda esta evidencia, los fondos estatales norteamericanos dirigidos a financiar la educación infantil descendieron \$30 millones durante 2009, un hecho sin precedentes, que hubieran disminuido más, si no hubiera sido por los fondos procedentes de la ley de la administración Obama, American Recovery and Reinvestment Act (ARRA). Mientras la escolarización infantil de 4 años en programas financiados con fondos estatales aumentó del $14 \%$ en 2002, al 27\% en 2010, la inversión estatal por escolar disminuyó de \$4.719 en 2002, a \$4.296 en 2010 (BARNETT, et al., 2010). Paradójicamente, la inversión estatal en educación infantil se volvió a reducir en $\$ 60$ millones durante 2010, el doble que el año anterior, pese a la inyección de $\$ 127$ millones procedentes de la ley federal American Recovery and Reinvestment Act (ARRA). Durante la última década, la financiación estatal de la educación infantil se dobló hasta alcanzar los \$5.100 millones en 2012, pero el número de escolares en programas estatales creció más rápidamente que estos fondos (BARNETT, et al., 2011). La mayoría de estados ofrecen educación infantil subvencionada. Los estados de Oklahoma, Georgia, Illinois, Nueva York, Florida, Virginia Occidental, Iowa y Washington (en un futuro cercano) poseen programas de educación preescolar de 4 años universal y voluntaria, con mayor o menor compromiso (gratuita para un número variante de niños) (ACKERMAN, et al., 2009). Podríamos, por tanto, resumir los beneficios de una educación infantil de calidad en los puntos que se presentan a continuación. No obstante, dichos logros sólo se podrán conseguir si el programa de educación infantil es verdaderamente de calidad (profesorado altamente cualificado y 
preparado, ratios reducidas, programas efectivos, etc.) y se combina con visitas a domicilio y un seguimiento tanto alimentario, como médico para los niños en riesgo, ya que la formación académica, no logrará sus objetivos, sin el apoyo y estímulos necesarios:

- Ahorros en la educación obligatoria (reducción de clases de refuerzo, repetición de curso, etc.).

- Aumento de los ingresos (tanto para los padres, como para los niños escolarizados y para la sociedad).

- Aumento de la recaudación tributaria (al aumentar la población empleada y sus retribuciones).

- Ahorro en programas sociales.

- Ahorro en el sistema penitenciario.

- Ahorros tangibles e intangibles por un descenso de la delincuencia.

- Ahorro por la disminución de la violencia y abuso al menor.

- Ahorro en programas sociales para la infancia.

- Ahorro en gasto sanitario.

- Ahorro familiar en la educación infantil.

- Reducción del fracaso escolar.

- Beneficios físicos, cognitivos, afectivos, que repercutirán en el bien estar de las generaciones futuras.

Como se desprende de la Tabla 1 en la página siguiente, la composición de la población norteamericana sigue cambiando, estimando que para 2050 las minorías superarán a la población blanca. El peso relativo de esta última etnia ha disminuido desde finales del siglo pasado un $20 \%$, en claro contraste con la evolución de la población hispana, que ha crecido un $152 \%$ y la asiática, un 200\%. El mayor retroceso de la población blanca la registra el segmento de población infantil y juvenil, debido a la disminución de la natalidad. Estos cambios demográficos requieren de compromisos públicos concretos y firmes para mejorar el nivel académico actual de las minorías, muy por debajo del de la población de etnia blanca, al revelar las estadísticas, que las diferencias académicas no han disminuido con el paso del tiempo. En 2012, la tasa de fracaso escolar se situaba en el 12,7\% para los jóvenes hispanos, frente al 6,6\% para el conjunto de jóvenes con edades comprendidas entre los 16 y 24 años (U.S. DEPARTMENT OF EDUCATION, 2013, Tabla 210.70). Por su parte, las evaluaciones externas de 2013 (U.S. DEPARTMENT OF EDUCATIN, 2013, Tablas 221.50 y 222.70) realizadas a los escolares de $4^{\circ}$ de primaria en la materia de lengua, indicaron que los escolares de etnia blanca de este curso académico habían obtenido una puntuación media de 231 putos, frente a 205 obtenidos por los alumnos de etnia negra y 207, por los hispanos. En el $8^{\circ}$ curso, los resultados fueron parecidos en la materia de matemáticas, los escolares de etnia blanca obtuvieron una puntuación media en dicha materia de 293 puntos, frente a 263, para los alumnos de etnia negra, y a 271, para los escolares hispanos. Por tanto, la educación infantil de calidad se ha 
convertido en una urgencia para garantizar el competitividad y cohesión social norteamericana durante el siglo XXI, como apuntan todos los expertos.

Tabla 1. Evolución del peso relativo de las principales etnias en la población norteamericana, por edades

\begin{tabular}{|c|c|c|c|c|}
\hline AÑO & Blancos & Negros & Hispanos & Asiáticos \\
\hline \multicolumn{5}{|c|}{ TOTAL } \\
\hline 1980 & 79,7 & 11,5 & 6,5 & 1,6 \\
\hline 1990 & 75,6 & 11,8 & 9,0 & 2,8 \\
\hline 1995 & 73,0 & 12,2 & 10,6 & 3,5 \\
\hline 2000 & 69,4 & 12,2 & 12,6 & 3,7 \\
\hline 2003 & 67,6 & 12,2 & 13,8 & 4,1 \\
\hline 2004 & 67,1 & 12,2 & 14,2 & 4,2 \\
\hline 2005 & 66,5 & 12,2 & 14,6 & 4,3 \\
\hline 2006 & 66,0 & 12,2 & 14,9 & 4,4 \\
\hline 2007 & 65,4 & 12,3 & 15,3 & 4,5 \\
\hline 2008 & 64,8 & 12,3 & 15,7 & 4,6 \\
\hline 2009 & 64,3 & 12,3 & 16,1 & 4,7 \\
\hline 2010 & 63,8 & 12,3 & 16,4 & 4,8 \\
\hline \multicolumn{5}{|c|}{$<4$ años } \\
\hline 1980 & 72,4 & 14,7 & 10,2 & 1,9 \\
\hline 1990 & 67,7 & 15,0 & 13,2 & 3,1 \\
\hline 1995 & 63,3 & 15,5 & 16,5 & 3,7 \\
\hline 2000 & 58,7 & 14,4 & 19,5 & 3,6 \\
\hline 2005 & 54,5 & 13,6 & 23,1 & 4,2 \\
\hline 2009 & 51,3 & 13,7 & 25,2 & 4,4 \\
\hline 2010 & 50,8 & 13,8 & 25,4 & 4,4 \\
\hline \multicolumn{5}{|c|}{ 5-17 años } \\
\hline 1980 & 74,6 & 14,5 & 8,5 & 1,7 \\
\hline 1990 & --- & --- & --- & --- \\
\hline 1995 & --- & --- & --- & --- \\
\hline 2000 & 62,0 & 15,0 & 16,4 & 3,4 \\
\hline 2005 & 58,5 & 14,9 & 19,0 & 3,8 \\
\hline 2009 & 55,4 & 14,3 & 21,7 & 4,2 \\
\hline 2010 & 54,7 & 14,2 & 22,4 & 4,4 \\
\hline \multicolumn{5}{|c|}{ 18-24 años } \\
\hline 1980 & 77,3 & 12,9 & 7,6 & 1,6 \\
\hline 1990 & --- & --- & --- & --- \\
\hline 1995 & --- & --- & --- & --- \\
\hline 2000 & 61,9 & 13,8 & 17,5 & 4,2 \\
\hline 2005 & 60,3 & 13,9 & 18,4 & 4,6 \\
\hline 2010 & 57,2 & 14,4 & 20,2 & 4,9 \\
\hline \multicolumn{5}{|c|}{ > 24 años } \\
\hline 1980 & 83,0 & 9,8 & 5,2 & 1,6 \\
\hline 1990 & 79,2 & 10,3 & 7,2 & 2,6 \\
\hline
\end{tabular}




\begin{tabular}{|l|l|l|l|l|}
1995 & 76,9 & 10,7 & 8,5 & 3,3 \\
2000 & 73,7 & 10,9 & 10,1 & 3,7 \\
2005 & 71,0 & 11,1 & 11,8 & 4,4 \\
2010 & 68,5 & 11,3 & 13,4 & 4,9 \\
\hline
\end{tabular}

*Fuente: (U.S. Department of Education, 2012:44)

\section{POBLACIÓN INFANTIL NORTEAMERICANA ESCOLARIZADA EN PROGRAMAS DE CALIDAD}

A raíz del reto lanzado por el presidente Barak Obama en 2009 de incrementar el número de ciudadanos norteamericanos con titulaciones de educación superior, varios expertos se han adherido a esta iniciativa, aportando sus opiniones, investigaciones y recomendaciones para lograr tal fin. Entre ellos se encuentra la asociación College Board Advocacy (LEE et al., 2011) nacida en 1900, quien formó una comisión en 2008 para profundizar en la mejora de la educación superior. El informe, publicado en 2011, demandaba un mayor compromiso público para financiar la educación infantil de calidad, por asentar los cimientos del éxito en educación superior, revelando los siguientes datos:

- $\quad$ El 34,4\% de la población infantil de 3 años se encontraba escolarizada en 2008.

- Entre los niños de 3 y 4 años, sólo el 47,5 \% formaba parte del sistema académico en 2008 (Tabla 2).

- La escolarización de la población infantil de 4 años alcanzó el 60,7\% ese mismo año.

Tabla 2. Porcentajes de niños norteamericanos con edades comprendidas entre los 3 y 4 años escolarizados clasificados por etnia. 2008

\begin{tabular}{|l|l|}
\hline ETNIA & $\%$ \\
\hline ASIATICOS & $53,30 \%$ \\
\hline NEGROS & $52,50 \%$ \\
\hline BLANCOS & $49,80 \%$ \\
\hline MEDIA & $\mathbf{4 7 , 5 0 \%}$ \\
\hline INDIOS & $45,00 \%$ \\
\hline HISPANOS & $38,50 \%$ \\
\hline
\end{tabular}

* Fuente: (LEE, et ál, 2011)

Una educación infantil de calidad se define como aquella impartida por profesionales altamente cualificados (con una licenciatura o grado en educación infantil y una óptima preparación y experiencia) y un bajo número de escolares por profesor, que se traduce en 1 profesor por cada 10 niños entre 3 y 4 años, con un máximo de 20 niños por aula (NIEER, 2013). La figura del maestro encargado del aula y la de su ayudante facilitan reducir esta relación y abaratar costes, ya que las remuneraciones que percibe un profesor auxiliar son inferiores. A estos dos requisitos debemos añadirle un programa educativo eficaz, estimulante, adaptado a la edad correspondiente y que potencie las cualidades 
individuales. El informe continuaba señalando que la financiación pública de este tramo educativo alcanzó al 25,4\% de la población infantil de 4 años en 2009. Dicho porcentaje se redujo hasta el 14,6\% cuando se incluye también a los párvulos de 3 años y descendió hasta el 3,7\%, para los escolares de 3 años, porcentajes bajos que dejan a muchos niños de esas edades sin escolarizar. Consecuentemente, la comisión recomendaba que los distritos y los consejos escolares locales cedieran instalaciones para tal fin y diseñaran programas de educación infantil de calidad con la finalidad de preparar a la población infantil para las etapas educativas posteriores.

El distrito de Washington DC escolariza al 84,7\% de su población infantil de 4 años, acercándose a la escolarización universal y al 68,5\% de su población infantil de 3 y 4 años, colocando a la capital federal a la cabeza, mientras que el estado de Nevada con $35,6 \%$ de los niños de 4 años y 27,6\% de los niños de 3 y 4 años, se sitúa en el último lugar de la escolarización de la población infantil. No es coincidencia que el estado de Nevada se encuentre a la cola en nivel académico de su población, frente a la capital federal, que se sitúa en el primer puesto en población cursando estudios superiores. Dicha Comisión apelaba a la urgencia de una educación preescolar voluntaria de calidad para que los niños desarrollen las habilidades necesarias para tener éxito escolar, especialmente los pertenecientes a clases menos afortunadas, que son los que más lo necesitan y son los menos escolarizados en programas de calidad.

\section{INICIATIVAS SOBRE EDUCACIÓN INFANTIL DE CALIDAD}

El programa financiado con fondos públicos Head Start (Comenzar con Ventaja) (2014), muy alabado por todas las administraciones federales norteamericanas, realiza una importante labor entre la infancia desaventajada, pero resulta claramente insuficiente, porque atiende sólo al 10\% de los niños de 4 años. Este porcentaje se reduce hasta el 8,6\%, para los niños de 3 y 4 años y hasta el 7,1\%, para los niños de 3 años:

El programa Early Head Start proporciona servicios fundamentales de desarrollo, de educación y de salud a mujeres embarazadas, a niños hasta los cinco años de edad para mejorar sus perspectivas de escolarización y empleo a largo plazo y a familias de rentas bajas. Los programas incluyen un fuerte enfoque en servicios sociales y en la participación de los padres, quienes reciben formación para cuidar y relacionarse de manera eficaz con sus hijos dentro del seno familiar y dentro de la comunidad. El objetivo general del programa Head Start y Early Head Start reside en mejorar la preparación de los niños para la escolarización obligatoria y ayudar a que las familias menos favorecidas sean autosuficientes.

Head Start: Continuación del programa anterior, está supervisado asimismo por Department of Health and Human Services (Departamento o ministerio de sanidad y de 
servicios sociales) para niños de edades comprendidas entre los 3 y 5 años (aunque algunos estados también lo ofrecen a niños más pequeños), pertenecientes a familias de rentas bajas. Los niños que asisten al programa Head Start participan en una amplia variedad de actividades educativas, al mismo tiempo, reciben atención médica y dental gratuitas y tienen cubierta su alimentación diurna, ofreciendo asimismo servicios para atender a escolares con necesidades especiales. Los escolares realizan actividades que les ayudan a desarrollarse tanto intelectualmente, como socialmente, emocionalmente y físicamente, a través del apoyo afectivo y formativo del personal, quien trabaja en estrecha colaboración con los padres. Los niños del programa Head Start aprenden a socializarse entre ellos, a resolver problemas, a compartir y vivir experiencias que les ayuden a adquirir confianza en sí mismos, aprendiendo a su vez, buenos hábitos y mejorando sus habilidades sociales, comunicativas y cognitivas.

El estado de Carolina del Norte (SMART START, 2011) fue agraciado con fondos federales procedentes del programa de la Adminstración Obama, Race To the Top-Early Learning Challenge Fund, en reconocimiento a su trayectoria en este tramo educativo. Hace apenas 20 años, dicho estado poseía uno de los índices de mortalidad infantil más elevado de EEUU, contando con los peores niveles de educación infantil, y con tan sólo el $33 \%$ de su población infantil en programas escolares de calidad. Hoy en día, los índices de mortalidad infantil han disminuido drásticamente, posee niveles académicos que la sitúan en los primeros puestos del ranking estatal norteamericano y el 66\% de su población infantil está escolarizada en programas de calidad, aunque no estatales. El objetivo del estado es escolarizar a la totalidad de su población infantil en programas escolares de calidad.

El estado de Oklahoma (OKLAHOMA STATE DEPARTMENT OF EDUCATION, 2012) implantó en 2005 la educación infantil universal y voluntaria de 4 años y en 2011 la escolarización obligatoria para los niños de 5 años. Este estado fue pionero en desarrollar programas de educación infantil. El 99\% de los distritos escolares de Oklahoma ofrece educación infantil pública de calidad para niños de 4 años, atendiendo a 38.441 escolares de esa edad, que representan el $75 \%$ de este colectivo y un $39 \%$ de los distritos de Oklahoma colaboran con una entidad privada en educación infantil. El número de alumnos por profesor (con titulación universitaria) es de 10 niños de 4 años por maestro. La iniciativa Early Childhood Pilot Program, nacida en 2005 y financiada entre la fundación privada George Kaiser Family Foundation, con una aportación de \$15 millones, y el estado con una aportación de 10 millones, escolariza a 2.000 niños de 0-3 años en riesgo en programas de calidad. El programa estatal Oklahoma Parents as Teachers (OPAT) (los padres son los profesores), financiado en 2010 con 2 millones de dólares, opera desde 1992, atendiendo a 4.500 niños y a sus padres, desde el embarazo hasta que cumplen 3 años, consistiendo en visitas periódicas a domicilio para ayudar y orientar a los padres en la educación y crianza de sus hijos. El programa SoonerStart (Comenzar más 
pronto) atiende a niños con minusvalías o problemas cognitivos, desde el nacimiento hasta los 3 años. Por su parte, la fundación George Kaiser Foundation (2012) promueve y financia el programa Tulsa Educare, dirigido a ayudar y escolarizar a niños bajo el umbral de la pobreza, desde los 6 meses hasta los 5 años. De hecho, el compromiso de dicha fundación con la educación infantil comienza con la financiación de las titulaciones universitarias de educación infantil de 2 años de duración en Tulsa Community College y de 4 años en University of Oklahoma Tulsa, al existir la necesidad de formar personal docente preparado para esta etapa educativa. Estas iniciativas han dado tan buenos resultados que el resto de estados está estudiando la posibilidad de implantar colaboraciones con fundaciones privadas en sus jurisdicciones. Según la fundación George Kaiser Foundation, dichos programas son la única vía para poder romper el círculo vicioso de la pobreza.

\section{CONCLUSIONES Y RETOS}

Pese a la aplastante evidencia de la importancia de la educación infantil de calidad en la formación de la ciudadanía, las administraciones estatales y federales norteamericanas son reacias a darle su apoyo económico, debido a que los frutos de dichas inversiones los recogerán sus sucesores. El fracaso escolar, el principal cuello de botella para el crecimiento personal, social y económico en EEUU, tiene sus orígenes en la carencia de una educación infantil de calidad. Paralelamente, los problemas relacionados con la salud, la delincuencia, el desempleo, etc., así como las desigualdades socioeconómicas se resolverían, en un alto porcentaje de situaciones, escolarizando a la población infantil en programas educativos de calidad, acompañados de visitas domiciliarias para aquellas familias en riesgo. La composición de la población norteamericana en las próximas décadas se decantará por las minorías, quienes ocuparán el grueso de la misma y quienes poseen niveles académicos deficientes y altas tasas de fracaso escolar. Por esta razón, cada vez son más las voces que se unen para solicitar una educación infantil universal de calidad, una propuesta recogida en el programa electoral de la primera legislatura del actual presidente Obama, pero que la reciente recesión económica y la pasividad de las administraciones públicas truncó. Los expertos coinciden en señalar que la educación infantil debe ser de calidad para que sea efectiva, la cual se caracteriza por el reducido número de alumnos por maestro (1:10) y por poseer profesorado altamente cualificado (en posesión de una licenciatura en educación infantil y una buena preparación). Tal como indica la fundación George Kaiser Foundation, dichos programas son la única vía para poder romper el círculo vicioso de la pobreza. En España el número de estudiantes por profesor durante la etapa de educación infantil es excesivamente elevado para poder impartir una educación infantil de calidad. La lejanía de los frutos cosechados por dicha etapa educativa es también en nuestro país factor disuasorio para movilizar a los agentes implicados en el desarrollo de una educación verdaderamente útil para la infancia. Un buen profesor, clave en la efectividad de la enseñanza, unido a la figura del maestro auxiliar o ayudante (modelo extendido por 
toda la geografía norteamericana) podría abaratar costes y potenciar la calidad en esta etapa educativa, que es fundamental para el éxito académico y personal de los ciudadanos.

\section{REFERENCIAS BIBLIOGRÁFICAS}

ACKERMAN, D. J., BARNETT, S., HAWKINSON, L. E. BROWN, K. K. y McGONIGLE, E. A. (2009): Providing Preschool Education for All 4-Year-Olds: Lessons from Six State Journeys. NIEER. (http://nieer.org/resources/policybriefs/19.pdf), consultado el 19 de Julio de 2012.

BARNETT, W. S., CAROLAN, M. E., FITZGERALD, J., SQIRES, J., $\quad$ H. (2011): The State of Preschool 2011. Yearbook. NIEER. 4. (http://nieer.org/sites/nieer/files/2011yearbook.pdf), consultado el 20 de julio de 2012.

BARNETT, W. S., EPSTEIN, D. J., CAROLAN, M. E., FITZGERALD, J., ACKERMAN, D. J. y FRIEDMAN, A. H. (2010): The State of Preschool 2010. Yearbook. NIEER. p.4. (http://nieer.org/sites/nieer/files/yearbook.pdf), consultado el 21 de julio de 2012.

BARTIK, T. (2006) J.: The Economic Development Benefits of Universal Preschool Education Compared to Traditional Economic Development Programs. Working paper. (Kalamazoo, MI, Upjohn Institute for Employment Research) (http://research.upjohn.org/cgi/viewcontent.cgi?article=1044\&context=reports), consultado el 21 de mayo de 2012.

BELFIELD, C. R. y SCHWARTZ, H. (2006): The Economic Consequences of Early Childhood Education on the School System. (New Brunswick. NJ, National Institute for Early Education Research). (http://nieer.org/resources/research/economic-consequences.pdf), consultado el 21 de abril de 2012.

CAMPBELL, F. A. y RAMEY, C. T. (2007): Carolina Abecedarian Project 7. American Psychological Association. (http://earlychildhoodrc.org/events/presentations/campbell-ramey.pdf), consultado el 8 de abril de 2012.

CENTER ON THE DEVELOPING CHILD (2011): Harvard University: Brain Architecture (http://developingchild.harvard.edu/key_concepts/brain_architecture/), consultado el 17 de febrero de 2014.

CLEMENT, D. (2005): Interview with James Heckman. Nobel prize-winning economist James Heckman on discrimination, job training and early childhood education. The Federal Reserve Bank of Minneapolis.

(http://www.minneapolisfed.org/publications_papers/pub_display.cfm?id=3278), consultado el 18 de junio de 2012. 
DICKENS, W. T., SAWHILL, I. y TEBBS, J. (2006): The Effects of Investing in Early Education on Economic Growth. (Washington DC, The Brookings Institution) (http://www.brookings.edu/views/papers/200604dickenssawhill.pdf), consultado el 10 de mayo de 2012.

GILLIAN, W. S. y ZIGLER, E. F. (2004): State Efforts to Evaluate the Effects of PreKindergarten. Yale University Child Study Center. (http://nieer.org/resources/research/StateEfforts.pdf), consultado el 6 de abril de 2012.

HEAD START (2014): An office of Administration for Children and families. Early Childhood learning and knowledge Center (ECLKC).

(http://eclkc.ohs.acf.hhs.gov/hslc/hs), consultado el 13 de marzo de 2012.

HECKMAN, J., J. (2012): The Economics of Human potential. The Heckman Equation. (http://www.heckmanequation.org/heckman-equation), consultado el 25 de junio de 2014.

HECKMAN, J. J. y MASTEROV, D. V. (2004): The Productivity Argument for Investing in Young Children. (Washington, DC, Committee for Economic Development) (http://jenni.uchicago.edu/Invest/FILES/dugger_2004-1202_dvm.pdf), consultado el 11 de abril de 2012.

LEE, J. M. Jr., et ál (2011): The College Completion Agenda 2011 Progress Report. College Board. Advocacy and Policy Center, pp. 17-42 (http://leadingsuccess.org/sites/default/files/7.pdf), consultado el 25 de marzo de 2012.

LYNCH, R. (2007): New Science Provides Compelling Framework for Early Childhood investment. Center on the Developing Child. Harvard University. (http://www.nursefamilypartnership.org/resources/files/PDF/ChildPolicyFrame work2007.pdf) (http://www.developingchild.harvard.edu/), consultado el 15 de febrero de 2012.

MUELLER, S., et ál (1974): Chicago Child-Parent Center. A Systematic Program of Effective Compensatory Education. Chicago.

(http://blogs.tc.columbia.edu/transitions/files/2010/09/15.Illinois Chicago-ChildParent-Center_profile_pdf), consultado el 6 de abril de 2012.

NIEER- THE NATIONAL INSTITUTE FOR EARLY EDUCATION RESEARCH (2013): The State Preschool Yearbook. 2013. Rutgers. p. 7 (http://nieer.org/sites/nieer/files/yearbook2013.pdf), consultado el 15 de noviembre de 2014.

OKLAHOMA STATE DEPARTMENT OF EDUCATION: Oklahoma Early Childhood Programs 2011 State Report. (http://www.ok.gov/sde/early-childhood-and-familyeducation), consultado el 5 de marzo de 2013. 
PROMISING PRACTICES NETWORK: The Chicago Child-Parent Centers (http://www.promisingpractices.net/program.asp?programid=98), consultado el 5 de abril de 2012.

REYNOLS, Arthur (2011): Early Childhood Education Program Yields High Economic Returns. College of Education and Human Development. University of Minnesota. (http://www.sciencedaily.com/releases/2011/02/110204091258.htm), consultado el 8 de abril de 2012.

REYNOLS, A. J. (2011): Large-Scale Early Education Linked to Higher Living Standards and Crime Prevention 25 Years Later. Science Daily. (http://www.sciencedaily.com/releases/2011/06/110609141556.htm), consultado el 18 de mayo de 2012.

REYNOLS, A. J. TEMPLE, J. A. OU, Suh-Ruu ARTEGA, I. A. y WHITE, B. A. (2011): School-Based Early Childhood Education and Age-28 Well- Being:

Effects by Timing, Dosage, and Subgroups. University of Minnesota. (http://www.cehd.umn.edu/icd/research/cls/docs/Science2011accepted.pdf http://www.cehd.umn.edu/ICD/cls/docs/Science2011accepted.pdf http://leadershiplinc.illinoisstate.edu/researchcompendium/documents/Scho olbasedEarlyChildhoodReynolds.pdf), consultado el 18 de junio de 2012.

REYNOLDS, A., TEMPLE, J. A, ROBERTSON, D. L. y MANN, E. A. (2001): Age 21 Cost-Benefit Analysis of the Title I Chicago Child-Parent Center Program. (http://www.waisman.wisc.edu/cls/cbaexecsum4.html), consultado el 8 de abril de 2012.

REYNOLS, A. (1999): Chicago Longitudinal Study. A Study of Children in the Chicago Public Schools. University of Minnesota. (http://www.cehd.umn.edu/icd/CLS/http://www.cehd.umn.edu/icd/cls/docs/clsweb. pdf), consultado el 5 de abril de 2012.

ROLNICK, A. y GRUNEWALD, R. (2003): Early Childhood Development: Economic Development with a High Public Return. The Federal Reserve Bank of

Minneapolis.

(http://minneapolisfed.org/publications papers/pub display.cfm?id=3832), consultado el 26 de mayo de 2012.

SCHWEINHART, L. J., BARNETT, W. S., BELFIELD, C. R., $\quad$ NORES, M. (2006): The High/Scope Perry Preschool Program: Cost- Benefit Analysis Using Data from the Age-40 Followup. (Volumen 41 no. 1). Journal of Human Resource. (http://www.jstor.org/discover/10.2307/40057261 ?uid=3737952\&uid=2129\&uid=2 \&uid=70\&uid=4\&sid=21101990885331), consultado el 10 de abril de 2012.

SCHWEINHART, L. J., MONTIE, J., XIANG, Z., BARNETT, W. S., BELFIELD, C. R., NORES, M. (2005): The High/Scope Perry Preschool Study Through Age 40 Summary, Conclusions, and Frequently Asked Questions. (Ypsilanti, MI, HighScope Press), pp. 1-3. 
(http://www.highscope.org/file/Research/PerryProject/specialsummary_rev201102 2.pdf), consultado el 9 de abril de 2012.

SMART START (2011): North Carolina Wins Race to the Top Early Learning Challenge Grant. (http://www.smartstart.org/tag/race-to-the-top), consultado el 5 de marzo de 2013.

THE CAROLINA ABECEDARIAN PROJECT: The Abecedarian Project. (http://abc.fpg.unc.edu), consultado el 7 de abril de 2014.

THE GEORGE KAISER FAMILY FOUNDATION (2012): Early Childhood Learning (http://www.gkff.org/areas-of-focus/education/early-childhood-learning.html), consultado el 5 de marzo de 2013.

THE RAND CORPORATION (2005): The Economics of Investing in Universal Preschool Education in California. (Santa Mónica, Ca,). (http://www.rand.org/pubs/monographs/2005/RAND_MG349.1.pdf), consultado el 18 de abril de 2012.

U. S. DEPARTMENT OF EDUCATION (2013). NCES: Digest of Education 2013. s: $106,10,106,20,203,10,210.70,221.50$ y $222.70,308,10$.

(http://nces.ed.gov/programs/digest/2013menu_tables.asp), consultado el 20 de noviembre de 2014.

U. S. DEPARTMENT OF EDUCATION (2012): NCES: Digest of Education 2011. p. 44. Tabla: 21. (http://nces.ed.gov/programs/digest/d11/tables/dt11_021.asp), consultado el 25 de febrero de 2013.

\section{PROFESIOGRAFÍA}

\section{Mercedes Varona Alabern}

Doctora en Ciencias de la Educación por la Universidad de Valencia, licenciada en Ciencias Empresariales por la Universidad de Barcelona y Certified Public Accountant (auditora) por el estado de Ohio (EEUU). Sus campos de investigación son la educación en EEUU: sus retos, novedades y tendencias y la relación de la economía en la educación. Ha trabajado principalmente en el sector empresarial. Datos de contacto: Departamento de Educación Comparada e Historia de la Educación. Facultad de Filosofía y Ciencias de la Educación. Universidad de Valencia. Email: varonamercedes@ gmail.com.

Fecha de recepción: 9 de noviembre de 2014.

Fecha de aceptación: 22 de febrero de 2015. 